\title{
NEW COMPUTABLE ENTANGLEMENT MONOTONES FROM FORMAL GROUP THEORY
}

\author{
JOSE CARRASCO, GIUSEPPE MARMO, AND PIERGIULIO TEMPESTA
}

\begin{abstract}
We present a mathematical construction of new quantum information measures that generalize the notion of logarithmic negativity. Our approach is based on formal group theory. We shall prove that this family of generalized negativity functions, due their algebraic properties, is suitable for studying entanglement in many-body systems.

Under mild hypotheses, the new measures are computable entanglement monotones. Also, they are composable: their evaluation over tensor products can be entirely computed in terms of the evaluations over each factor, by means of a specific group law.

In principle, they might be useful to study separability and (in a future perspective) criticality of mixed states, complementing the role of Rényi's entanglement entropy in the discrimination of conformal sectors for pure states.
\end{abstract}

\section{Contents}

1. Introduction

2. Groups and entropies: a general approach

2.1. The Composability Axiom

2.2. Group logarithms

2.3. Group entropies

3. Negativity and PPT operations

4. $p$-norm group negativities 8

4.1. Definitions 8

4.2. Main result 10

5. Trace-norm group negativities and monotonicity 11

6. p-norm additive negativity as an upper bound for distillability 12

7. Future Perspectives 14

Acknowledgement $\quad 15$

8. Appendix:Formal groups and formal rings $\quad 15$

$\begin{array}{ll}\text { References } & 16\end{array}$

\section{Introduction}

The study of entanglement of many-body systems represents one of the most relevant challenges of modern research in quantum physics, due to its intrinsic theoretical interest and its applicative relevance. In this context, the determination of

Date: February 08, 2021. 
suitable information measures, allowing one to detect the entanglement in complex quantum systems is of outmost importance. [3].

When analyzing compound systems made up of spatially separated parties that can communicate with each other, it is very common and natural to focus on protocols that consist on local operations assisted by classical communication (LOCC); they map the set of separable states into itself. Operations preserving the positivity of the density matrix after partial transposition (PPT-operations) are also of special relevance, since all LOCC are in particular PPT-protocols, which in turn map the set of states with positive partial transpose into themselves.

In order to design a quantum information measure $E$ as an entanglement detector, certain conditions should be satisfied regarding LOCC or PPT-protocols. A fundamental requirement is that $E$ should be non-increasing on average under LOCC or PPT-preserving operations $[26,17,19]$. Precisely, we require that

$$
E(\rho) \geqslant \sum_{i} p_{i} E\left(\rho_{i}\right)
$$

where each of the states $\rho_{i}$, in a given Hilbert space, is obtained with probability $p_{i}$ when some LOCC or PPT-operation is applied to $\rho$. Another desirable property is that $E$ should be able to discriminate whether a state is separable or not, or at least, whether a state belongs to the set of states invariant under PPT-operations (PPT-invariant). The last condition is weaker: indeed, this set contains the set of separable states; however, there are PPT-invariant states that are not separable (they are said to contain bound entanglement).

An entanglement monotone is a quantum measure satisfying both properties, namely it is non-increasing on average and it can discriminate the set of PPTinvariant or separable states.

The construction of quantum measures which fulfill these requirements and admit an explicit, analytic form is an interesting problem. Important examples are provided by the negativity and the logarithmic negativity, introduced in the seminal paper [43]. In particular, in [25] it was shown that the logarithmic negativity is an entanglement monotone.

In recent years, both the negativity and logarithmic negativity have been largely investigated as entanglement measures for mixed states [25, 27], as well as for their prominent role in several contexts of quantum field theory, in particular in the case of conformal field theories (CFTs) [11, 29, 6, 7].

As is well known, both Rényi and Tsallis entropies can detect criticality in some specific contexts when the von Neumann-Shannon entropy can not.

There are one-dimensional quantum systems whose von Neumann-Shannon entanglement entropy coincides with that of a CFT but their Rényi entanglement entropies do not (for all values of $n$ ). Consequently, instead of comparing their spectra (which would be the only definitive way of asserting that the quantum critical system is effectively described by a CFT) one can compute their Rényi entanglement entropies as a thinner criterion than the von Neumann-Shannon one (see also [8] for the relevance of Rényi's entropy in the study of multi-block entanglement entropy of free fermion systems). Since the standard (non-parametric) negativity has been recently computed for CFTs, our ultimate goal is to find a meaningful and computable parametric generalization of the negativity in order to provide eventually thinner and more specific criteria to classify universality classes of one-dimensional quantum critical systems. 
As a first step in this research program, the aim of this article is essentially of a mathematical nature, namely to establish a general mathematical framework that allows us to construct a new, large class of parametric quantum information measures playing the role of entanglement monotones (or quasi-monotones, as we will prove) for mixed states. More precisely, we will show that a wide class of generalized entropic information functions can be defined by means of formal group theory. Due to the fact that these new group-theoretical functions widely generalize the notion of logarithmic negativity, we shall call them group negativities.

As we will show, from a technical point of view, group negativities are multiparametric concave functions (generalized logarithms), depending on the $p$-norm of the partial transposition of a quantum state. The trace-norm subclass is recovered when $p=1$ and is related to quantized versions of the group entropies proposed in [37], computed over partially transposed states. Moreover, if the generalized logarithm is chosen to be the standard one, we recover the original logarithmic negativity introduced in [43].

More specifically, the trace-norm subclass is composed by multi-parametric computable measures of entanglement. Indeed, as a consequence of Proposition 2 and under the hypotheses of validity of the Peres criterion [24], any trace-norm group negativity allows one to detect entanglement in mixed bipartite states: the strict positivity of the functional is sufficient to ensure that the state is entangled. We remind that Peres's criterion (positivity of the partial transpose of a state) is a necessary and sufficient condition for the separability of $2 \times 2$ and $2 \times 3$ systems, and is still necessary in higher dimensions [18]. For the trace-norm subclass of group negativities, our main results are the following:

(1) They are computable measures of entanglement and provide separability tests for bipartite mixed states.

(2) They are entanglement monotones.

(3) They are composable: each group negativity can be computed for a pure separable state in terms of the group negativities of each of its (non necessarily pure) reductions.

The composability property is guaranteed by the specific functional form of group negativities and could be important in the context of distillability. We remind that if we have multiple copies of a bipartite state $\rho$, its distillation rate is the best ratio between the number of maximally entangled pairs which can be obtained from it (distilled) by means of some LOCC and the number of copies of the original state needed. The group negativity of the $n$ copies of $\rho$ can be expressed through the group negativity of $\rho$.

Besides, as we shall see, generalized negativities constructed with the standard logarithm and $p$-norms with $p>1$ ( $p$-norm group negativities) are potentially interesting as well, since they represent auxiliary information tools that could be useful to determine bounds to distillability rates for distillation processes, in different scenarios. We observe that the simplest $p$-norm group negativity (see Eq. (11) below) is associated to the additive formal group law (that is to say, the generalized logarithm is the standard one) for all $p \geqslant 1$ as in Definition 7; we shall prove in Theorem 3 that they provide upper bounds for the entropy of distillation.

Concerning genuine $p$-norm group negativities, our main result, obtained under mild conditions, is that they are quasi-monotones in the sense that their increasing after an LOCC (or trace-preserving CP-PPT) operation is bounded on average by 
a non-negative function $k(p)$ independent of the state considered. This function can be made arbitrarily small, in the limit $p \rightarrow 1$.

From a mathematical point of view, the construction of group negativities relies on the theory of formal groups $[4,16]$, which represents an important branch of algebraic topology, with many applications in combinatorics and number theory (see e.g. [33, 35]). According to the arguments exposed above, we consider the composability property essential in order to discuss distillability, since the underlying formal group law controls how the available information is redistributed when independent subsystems are combined into a new one.

The article is organized as follows. In Section 2, we shall review briefly the basic aspects of formal group theory, with special emphasis on its role in the theory of generalized entropies. In Section 3, the notion of negativity is discussed. In Section 4, the main definitions of the class of $p$-norm negativity are introduced and their main properties are proved. The subclass of the trace-norm group negativities is discussed in Section 5. An additive $p$-norm is introduced in Section 6. Some open problems and future perspectives are discussed in the final Section 7 .

\section{Groups and entropies: a general approach}

We shall start by reviewing some aspects of the group-theoretical classification of generalized entropies; also, we shall describe how this approach can be used in our formulation of generalized negativities. Some definitions of formal group theory

will be presented in the Appendix (see also [16] for a thorough exposition, and [30] for a shorter introduction to the topic).

2.1. The Composability Axiom. The notion of composability, introduced in [41], has been put in axiomatic form in [36], [37], [39] and related to formal group theory via the notion of group entropy. We shall briefly discuss these concepts of composability and group entropies as in [36], [39] in order to illustrate the potential relevance of the group-theoretical machinery in the study of composite quantum systems. We also mention that the relevance of the notion of group entropy in classical information geometry has been elucidated in [28].

Let $\left\{p_{i}\right\}_{i=1, \cdots, W}$, with $W>1, \sum_{i=1}^{W} p_{i}=1$ be a discrete probability distribution; we denote by $\mathcal{P}_{W}$ the set of all discrete probability distributions with $W$ entries. Let $S$ be a real function defined on $\mathcal{P}_{W}$.

Definition 1. We shall say that $S$ is strictly composable if there exists a continuous function of two real variables $\Phi(x, y)$ such that the following properties are satisfied.

(C1) Composability: $S\left(P_{A} \times P_{B}\right)=\Phi\left(S\left(P_{A}\right), S\left(P_{B}\right)\right)$, where $A$ and $B$ are two arbitrary statistically independent systems with associated probability distributions $P_{A}$ and $P_{B}$, respectively.

(C2) Symmetry: $\Phi(x, y)=\Phi(y, x)$.

(C3) Associativity: $\Phi(x, \Phi(y, z))=\Phi(\Phi(x, y), z)$

(C4) Null-composability: $\Phi(x, 0)=x$

Observe that the mere existence of a function $\Phi(x, y)$ taking care of the composition process as in $(C 1)$ is necessary, but not sufficient to ensure that a given entropy may be suitable for information-theoretical or thermodynamic purposes: 
this function must satisfy all the requirements above to be admissible. Indeed, in general the entropy of the system compounded by the subsystems $A$ and $B$ should not vary if we exchange the labels $A$ and $B$, thus justifying condition (C2). In the same vein, condition (C3) guarantees the composability of more than two systems in an associative way, this property being crucial to define a zeroth law. Finally, condition (C4) in our opinion is also necessary since if we compound two systems $A$ and $B$ and the latter has zero entropy, then the total entropy must coincide with that of the former.

The set of requirements $(\mathrm{C} 2)-(\mathrm{C} 4)$ altogether represent the composability axiom, which replaces the additivity axiom in the set of the four Shannon-Khinchin axioms. These axioms, introduced by Shannon and Khinchin as conditions for an uniqueness theorem for the Boltzmann entropy, represent fundamental, non-negotiable requirements that an entropy $S[p]$ should satisfy to be physically meaningful: continuity with respect to all variables $p_{1}, \ldots, p_{W}$, maximization over the uniform distribution, expansibility (adding an event of zero probability does not affect the value of $S[p])$.

Definition 2. A group entropy is a function $S: P_{W} \rightarrow \mathbb{R}^{+} \cup\{0\}$ which satisfies the Shannon-Khinchin axioms (SK1)-(SK3) and the composability axiom (C1)-(C4).

Our construction of group negativities is inspired by this notion.

A function $\Phi(x, y)$ satisfying the properties $(\mathrm{C} 2)-(\mathrm{C} 4)$ is a formal group law. This is the origin of the connection between entropic measures and formal group theory (presented for the first time in [34]), as we shall illustrate in the forthcoming considerations. Among the examples of group entropies, we mention the class of $Z$-entropies, considered in [37].

We will show that the notion of logarithmic negativity can be generalized by means of a mathematical formalism based on formal group theory. Our main result is the following: there exists a "tower" of new, parametric information measures, each of them reducing to the logarithmic negativity in a certain regime.

Many other approaches to generalized entropies have been proposed in the literature. In particular, we mention the one developed in [20] (strongly pseudo-additive entropies) and the one advocated in [21], based on statistical inference theory. These two approaches as well as the group-theoretical allow to define from an independent perspective classes of entropies possessing many interesting properties.

2.2. Group logarithms. Our construction relies on the notion of group logarithm associated to every formal group law. There is a certain freedom concerning the regularity properties in its definition, depending on the application under consideration. The standard logarithm is associated to the additive formal group law.

Definition 3. A group logarithm is a strictly increasing and strictly concave function $\log _{G}:(0, \infty) \rightarrow \mathbb{R}$, with $\log _{G}(1)=0$ (possibly depending on a set of real parameters), satisfying a functional equation of the form

$$
\log _{G}(x y)=\chi\left(\log _{G}(x), \log _{G}(y)\right)
$$

where $\chi(x, y)$ fulfills the requirements $(C 2)-(C 4)$. This equation will be called the group law associated with $\log _{G}(\cdot)$.

An useful result is the following, simple proposition. 
Proposition 1. Let $G: \mathbb{R} \rightarrow \mathbb{R}$ be a strictly increasing function, vanishing at zero. The function $\Lambda_{G}(x)$ defined by

$$
\Lambda_{G}(x):=G\left(\ln x^{\gamma}\right), \quad x>0, \quad \gamma>0
$$

satisfies a functional equation of the form (2).

Proof. Observe that

$$
\begin{aligned}
\Lambda_{G}(x y) & =G\left(\ln x^{\gamma}+\ln y^{\gamma}\right)=G\left(G^{-1}\left(\Lambda_{G}(x)\right)+G^{-1}\left(\Lambda_{G}(y)\right)\right) \\
& =\chi\left(\Lambda_{G}(x), \Lambda_{G}(y)\right) .
\end{aligned}
$$

In other words, we have

$$
\chi(x, y)=G\left(G^{-1}(x)+G^{-1}(y)\right),
$$

which is indeed a group law.

We shall consider functions of the form $G(t)=t+O\left(t^{2}\right)$ for $t \rightarrow 0$, in such a way that $\chi(x, y)=x+y+\ldots$.

Remark 1. From now on, we shall focus on group logarithms of the form

$$
\log _{G}(x)=G(\ln x) .
$$

Here $G$ is a suitable, strictly increasing function (vanishing at 0 ), assuring concavity of $\log _{G}(x)$ (see [37] for a general discussion of sufficient conditions for $G$ to comply with these requirements).

The formal inverse of a group logarithm will be called the associated group exponential; it is defined by

$$
\exp _{G}(x)=e^{G^{-1}(x)}
$$

Remark 2. A first, relevant example of nontrivial group logarithm is given by the so called $q$-logarithm. We have

$$
G(t)=\frac{\mathrm{e}^{(1-q) t}-1}{1-q}, \quad \log _{q}(x)=G(\ln x)=\frac{x^{1-q}-1}{1-q}, \quad q>0 .
$$

This logarithm has been largely investigated in connection with nonextensive statistics [40], [41].

Concerning group exponentials, notice that when $G(t)=t$, we obtain the standard exponential; when as before $G(t)=\frac{e^{(1-q) t}-1}{1-q}$, we recover the $q$-exponential $e_{q}(x)=[1+(1-q) x]_{+}^{\frac{1}{1-q}}$, and so on.

Infinitely many other examples of group logarithms and exponentials are provided, for instance, in [34].

2.3. Group entropies. The general family of group entropies is usually defined in terms of generalized logarithms, which in turn allow us to realize the composition laws for the entropies in terms of formal group laws. We remind that a particular instance of the family of group entropies is the class of $Z$-entropies defined in [37] within the approach described above. Their general form, for $\alpha>0$, is

$$
Z_{G, \alpha}\left(p_{1}, \ldots, p_{W}\right):=\frac{\log _{G}\left(\sum_{i=1}^{W} p_{i}^{\alpha}\right)}{1-\alpha},
$$

where $\log _{G}$ is a group logarithm. We also mention that a widely generalized, relative-entropy version of this class, the $(h, f)$-divergence, has been introduced 
in [28], in the context of information theory. Indeed, given two probability distributions $P, Q$ such that $p_{i}, q_{i}>0, i=1, \ldots, W$, we can immediately deduce the relative $Z$-class:

$$
Z_{G, \alpha}(P \| Q):=\log _{G}\left[\left(\sum_{i=1}^{W} p_{i}^{\alpha} q_{i}^{1-\alpha}\right)^{\frac{1}{\alpha-1}}\right],
$$

$\alpha \neq 1$, which is nothing but a special version of the $(h, f)$-divergence, for $h(x)=$ $G\left(\ln x^{\gamma}\right), f(x)=x^{\alpha}, \gamma=\frac{1}{\alpha-1}$. Clearly, alternative classes are easily obtainable from the $(h, f)$-relative entropies of [28] by means of different choices of $h$ and $f$. To conclude this review, we recall that more general classes of group entropies have been defined in [28], [38] and [39].

In this section, the group-theoretical formalism necessary for the formulation of our main results has been established. From a conceptual point of view, the main motivation for introducing this approach relies in the following observation: As the logarithmic negativity is associated to the additive formal group law, so it is natural to define new entanglement measures associated to different, nonadditive composition laws by means of formal group theory.

\section{Negativity and PPT operations}

We introduce now the quantum formalism relevant in the forthcoming discussion. Let us denote by $\mathcal{B}_{1}$ and $\mathcal{B}_{2}$ the space of bounded linear operators of the Hilbert spaces $\mathcal{H}_{1}$ and $\mathcal{H}_{2}$ respectively. For a bipartite mixed state $\rho \in \mathcal{B}_{1} \otimes \mathcal{B}_{2}$, let us denote by $\rho^{\Gamma}$ its partial transposition with respect to $\mathcal{H}_{2}$ (the final result will not change if we choose $\mathcal{H}_{1}$ in this definition). The action of partial transposing is defined in the space $\mathcal{B}$ of bounded linear operators of the Hilbert space $\mathcal{H}=\mathcal{H}_{1} \otimes \mathcal{H}_{2}$ by extending (by linearity) the action over pure separable states $\sigma \otimes \tau \in \mathcal{B}$, with $\sigma \in \mathcal{B}_{1}$ and $\tau \in \mathcal{B}_{2}$ :

$$
(\sigma \otimes \tau)^{\Gamma}=\sigma \otimes \tau^{\mathrm{T}}
$$

where $\tau^{\mathrm{T}} \in \mathcal{B}_{2}$ is the transpose of $\tau$.

Definition 4. Given an element $A \in \mathcal{B}$, we introduce $|A|:=\{A\}_{+}-\{A\}_{-}$where $\{\cdot\}_{+}$and $\{\cdot\}_{-}$are its positive and negative parts, i.e., its restrictions to the eigenspaces of positive and negative eigenvalues respectively. The trace-norm $\|\cdot\|_{1}$ of an operator $A$ is defined as $\|A\|_{1}=\operatorname{tr}|A|$.

Note that $A=\{A\}_{+}+\{A\}_{-}$; if $A$ is Hermitian, then $\{A\}_{+}-\{A\}_{-}=\sqrt{A A^{\dagger}}$ where $\sqrt{B}$ represents any operator $C \in \mathcal{B}$ such that $C^{2}=B \in \mathcal{B}$.

Definition 5. Given a bipartite mixed state $\rho$, its negativity is defined to be the function $N(\rho):=\frac{1}{2}\left(\left\|\rho^{\Gamma}\right\|_{1}-1\right)$, while its logarithmic negativity is the function $L(\rho):=\ln \left\|\rho^{\Gamma}\right\|_{1}$.

The monotonicity of $L(\rho)$ was proved in [25]. Precisely, the inequality

$$
L(\rho) \geqslant \sum p_{i} L\left(\rho_{i}\right)
$$

holds, where $\rho_{i} \propto \mathcal{A}_{i}(\rho)$ is the normalized state associated to outcome $i$ after applying the trace-preserving completely positive operation $\mathcal{A}=\sum_{i} \mathcal{A}_{i}$. Note that $\mathcal{A}$ maps the set of PPT states into itself and also that the result of applying $\mathcal{A}$ to $\rho$ can be seen as an ensemble with elements $\rho_{i}$ appearing with probabilities 
$p_{i}=\operatorname{tr} \mathcal{A}_{i}(\rho)$. The logarithmic negativity $L$ is also an upper bound to distillable entanglement, as was shown in [43].

\section{4. $p$-NORM GROUP NEGATIVITIES}

4.1. Definitions. As before, we shall consider a composite quantum system, whose associated Hilbert space $\mathcal{H}$ has dimension $N$; we shall denote by $\mathcal{B}(\mathcal{H})$ the linear space of bounded linear operators on $\mathcal{H}$.

Consider the Scatten $p$-norms

$$
\|A\|_{p}=\left(\left(s_{1}(A)\right)^{p}+\cdots+\left(s_{N}(A)\right)^{p}\right)^{1 / p}, \quad p \geqslant 1
$$

for any $A \in \mathcal{B}$ with singular values $s_{i}(A)$; the limit $p \rightarrow \infty$ will be denoted by $\|\cdot\|_{\infty}$. We introduce now the main objects of our analysis.

Definition 6. The function $\mathcal{L}_{G, p}: \mathcal{B} \rightarrow \mathbf{R}$, defined for any state $\rho \in \mathcal{B}$ and $p \geqslant 1$ as

$$
\mathcal{L}_{G, p}(\rho):=\log _{G}\left\|\rho^{\Gamma}\right\|_{p}
$$

is said to be the p-norm group negativity of the state $\rho$. Here $\log _{G}(\cdot)$ is a group logarithm of the form (5).

Clearly, trace-norm group negativities are obtained when $p=1$. A simple but interesting, new case is the additive one, obtained when $\log _{G}(x)=\ln x$ for $p>1$.

Definition 7. The function

$$
\mathcal{L}_{p}(\rho)=\ln \left\|\rho^{\Gamma}\right\|_{p}, \quad p \geqslant 1
$$

will be called the logarithmic p-norm negativity of a mixed state $\rho$.

Remark 3. Obviously, in information-theoretical applications one could replace $\ln (x)$ with $\log _{2}(x)$ in Eq. (11) (as in the standard definition for $p=1$, by Vidal and Werner in [43]), without altering the main properties of the function.

We will show that the quantity $\mathcal{L}_{G, p}(\cdot)$ is bounded on average under LOCC (trace-preserving CP-PPT) operations. This bound can be made arbitrarily close to zero (and in particular is exactly zero in the limit $p=1$ ).

We shall first deal with a deterministic trace-preserving CP-PPT operation $\mathcal{A}$; then we shall consider a general, not necessarily deterministic operation, which maps a state $\rho$ into an ensemble of states $\rho_{i}=\mathcal{A}_{i}(\rho)$, each appearing with probability $p_{i}=\operatorname{tr} \mathcal{A}_{i}(\rho)$ (where each operation $\mathcal{A}_{i}$ is a CP-PPT operation and $\sum_{i} \mathcal{A}_{i}$ is trace-preserving). Let us regard the partial transposition as an operator $\Gamma: \mathcal{B} \rightarrow \mathcal{B}$, with $\Gamma(\rho)=\rho^{\Gamma}$. This operation is clearly involutive. We can define a linear map $\mathcal{A}^{\Gamma}: \mathcal{B} \rightarrow \mathcal{B}$ as $\mathcal{A}^{\Gamma}(\sigma):=\Gamma \circ \mathcal{A} \circ \Gamma(\sigma) ;$ equivalently, $\mathcal{A}^{\Gamma} \circ \Gamma=\Gamma \circ \mathcal{A}$.

We propose a simple Lemma, useful in the forthcoming discussion.

Lemma 1. Let $\mathcal{A}: \mathcal{B} \rightarrow \mathcal{B}$ be any PPT quantum operation. If $\mathcal{A}$ is positive and preserves the positivity of the partial transpose, then its partial transpose $\mathcal{A}^{\Gamma}$ is also positive.

Proof. Since $\mathcal{A}$ is a PPT operation, $\Gamma \circ \mathcal{A}(\rho)$ is positive if $\Gamma(\rho)$ is positive. Due to the fact that $\Gamma$ is an involution, writing $\sigma=\Gamma(\rho)$ and $\rho=\Gamma(\sigma)$ we conclude that $\Gamma \circ \mathcal{A} \circ \Gamma(\sigma)$ is positive if $\Gamma \circ \Gamma(\sigma)=\sigma$ is positive. The result follows from the relation $\mathcal{A}^{\Gamma}=\Gamma \circ \mathcal{A} \circ \Gamma$. 
The following statement is due to Plenio [25].

Lemma 2. Let $\mathcal{A}: \mathcal{B} \rightarrow \mathcal{B}$ be a trace-preserving completely positive operation. Then $\operatorname{tr}|\mathcal{A}(\rho)| \leqslant \operatorname{tr}|\rho|$.

Proof. Note that

$$
\{\mathcal{A}(\cdot)\}_{+}=\left\{\mathcal{A}\left(\{\cdot\}_{+}\right)+\mathcal{A}\left(\{\cdot\}_{-}\right)\right\}_{+} \leqslant \mathcal{A}\left(\{\cdot\}_{+}\right)
$$

due to the fact that $\mathcal{A}\left(\{\cdot\}_{-}\right)=-\mathcal{A}\left(-\{\cdot\}_{-}\right)$and $-\{\cdot\}_{-}$is positive or zero, so that by linearity $\mathcal{A}\left(\{\cdot\}_{-}\right)$is negative or zero. Also, observe that

$$
\begin{aligned}
|\mathcal{A}(\rho)|=\{\mathcal{A}(\rho)\}_{+}-\{\mathcal{A}(\rho)\}_{-} & =\{\mathcal{A}(\rho)\}_{+}+\{-\mathcal{A}(\rho)\}_{+} \\
& =\{\mathcal{A}(\rho)\}_{+}+\{\mathcal{A}(-\rho)\}_{+} \leqslant \mathcal{A}\left(\rho_{+}\right)+\mathcal{A}\left(\{-\rho\}_{+}\right) .
\end{aligned}
$$

Here we have used twice inequality (12). The result follows by noting that $\rho_{-}=$ $-\{-\rho\}_{+}$and taking into account linearity in the RHS of the last inequality to obtain $|\rho|=\rho_{+}-\rho_{-}$as the argument of $\mathcal{A}$.

Definition 8. A group logarithm $\log _{G}(x)$ such that

$$
\log _{G}(x y) \leqslant \log _{G} x+\log _{G} y
$$

will be said to be subadditive.

In order to prove the main results of this section, we state the following

Lemma 3. The inequality

$$
\sum_{i} p_{i}\left\|\rho_{i}^{\Gamma}\right\|_{p} \leqslant \operatorname{tr}\left|\rho^{\Gamma}\right|
$$

holds for $p \geqslant 1$ under trace-preserving CP-PPT operations.

Proof. Observe that

$$
\sum_{i} p_{i}\left\|\rho_{i}^{\Gamma}\right\|_{p}=\sum_{i}\left\|\mathcal{A}_{i}^{\Gamma}\left(\rho^{\Gamma}\right)\right\|_{p}=\sum_{i}\left(\operatorname{tr}\left|\mathcal{A}_{i}^{\Gamma}\left(\rho^{\Gamma}\right)\right|^{p}\right)^{1 / p}
$$

where $\left|\mathcal{A}_{i}^{\Gamma}\left(\rho^{\Gamma}\right)\right|$ is of course a positive operator. Also, for any positive operator $A$ we have that

$$
\operatorname{tr} A^{p} \leqslant(\operatorname{tr} A)^{p}, \quad p \geqslant 1
$$

Thus, we obtain

$$
\left(\operatorname{tr}\left|\mathcal{A}_{i}^{\Gamma}\left(\rho^{\Gamma}\right)\right|^{p}\right)^{1 / p} \leqslant \operatorname{tr}\left|\mathcal{A}_{i}^{\Gamma}\left(\rho^{\Gamma}\right)\right| \leqslant \operatorname{tr} \mathcal{A}_{i}^{\Gamma}\left(\left|\rho^{\Gamma}\right|\right)
$$

where the last inequality follows from Lemma 2. Therefore we have shown that

$$
\sum_{i} p_{i}\left\|\rho_{i}^{\Gamma}\right\|_{p} \leqslant \sum_{i} \operatorname{tr} \mathcal{A}_{i}^{\Gamma}\left(\left|\rho^{\Gamma}\right|\right)=\operatorname{tr}\left|\rho^{\Gamma}\right| .
$$




\subsection{Main result.}

Theorem 1. The group p-norm negativity $\mathcal{L}_{G, p}(\rho)=\log _{G}\left\|\rho^{\Gamma}\right\|_{p}$ associated with a subadditive group logarithm, for any $p \geqslant 1$ is bounded on average under tracepreserving CP-PPT operations, that is, there exists a constant $k(p)$ such that

$$
\sum_{i} p_{i} \mathcal{L}_{G, p}\left(\rho_{i}\right)-\mathcal{L}_{G, p}(\rho) \leqslant k(p) .
$$

Proof. Since by definition a group logarithm is a concave function, then

$$
\sum_{i} p_{i} \mathcal{L}_{G, p}\left(\rho_{i}\right)=\sum_{i} p_{i} \log _{G}\left\|\rho_{i}^{\Gamma}\right\|_{p} \leqslant \log _{G}\left(\sum_{i} p_{i}\left\|\rho_{i}^{\Gamma}\right\|_{p}\right) .
$$

Now, since $\log _{G}(x)$ is also a strictly increasing function, by means of Lemma 3, we deduce

$$
\sum_{i} p_{i} \mathcal{L}_{G, p}\left(\rho_{i}\right) \leqslant \log _{G} \operatorname{tr}\left|\rho^{\Gamma}\right|
$$

Let us denote by $N$ the dimension of the ambient Hilbert space $\mathcal{H}$. We can observe that

$$
\operatorname{tr}\left|\rho^{\Gamma}\right|=\left\|\rho^{\Gamma}\right\|_{1} \leqslant c(p)\left\|\rho^{\Gamma}\right\|_{p}
$$

where $c(p)=N^{1-1 / p}$, and $\rho \in \mathcal{B}(\mathcal{H})$.

Finally, due to subadditivity of $\log _{G}$, we have that

$$
\sum_{i} p_{i} \mathcal{L}_{G, p}\left(\rho_{i}\right) \leqslant \log _{G}\left\|\rho^{\Gamma}\right\|_{1} \leqslant \log _{G} c(p)+\log _{G}\left\|\rho^{\Gamma}\right\|_{p}
$$

To conclude, we introduce

$$
k(p):=\log _{G} c(p) .
$$

Thus, the previous inequality reduces to relation (17).

Remark 4. An interesting aspect of inequality (17) is that $k(p)$ can be made arbitrarily small by considering $p$-norms where $p=1+\delta$, with $\delta>0$ arbitrarily close to zero. Thus, for any $\epsilon>0$ there exists a value $\delta$ such that

$$
\sum_{i} p_{i} \mathcal{L}_{G, p}\left(\rho_{i}\right) \leqslant \mathcal{L}_{G, p}(\rho)+\epsilon
$$

Due to the latter property, we shall say that the $p$-norm negativity $\mathcal{L}_{G, p}(\rho)$ is an $\epsilon$-monotone (or quasi-monotone).

Remark 5. The hypotheses of Theorem 1 are actually satisfied by an infinite family of group logarithms. For instance, the group exponential and logarithm considered in Remark 2 possess all required properties: Indeed, $\log _{q}(x)$ is strictly concave, monotonically increasing and subadditive for $q>1$. In general, we have $\log _{q}\left(\rho^{X} \otimes \rho^{Y}\right)=\log _{q}\left(\rho^{X}\right)+\log _{q}\left(\rho^{Y}\right)+(1-q) \log _{q}\left(\rho^{X}\right) \log _{q}\left(\rho^{Y}\right)$. We introduce now the corresponding negativity measure.

Definition 9. The p-norm q-negativity for any $\rho \in \mathcal{B}$ is the function

$$
\mathcal{L}_{p}^{(q)}(\rho):=\frac{\left(\left\|\rho^{\Gamma}\right\|_{p}\right)^{1-q}-1}{1-q}, \quad q>1 .
$$

In summary, the class of subadditive $p$-norm group negativities, defined in this Section, share with the standard negativity the crucial property of being bounded on average, as assured by Theorem 1; this result represents one of the main contributions of this work. 


\section{TRACE-NORM GROUP NEGATIVITIES AND MONOTONICITY}

A particular case of the previous construction, interesting on its own, is the trace-norm class, corresponding to $p=1$ in the previous analysis.

Definition 10. A trace-norm group negativity $L_{G}: \mathcal{B} \rightarrow \mathbf{R}$, where $\mathcal{B}$ is the space of bounded linear operators of a Hilbert space $\mathcal{H}$, is the function

$$
L_{G}(\rho):=\log _{G}\left\|\rho^{\Gamma}\right\|_{1}, \quad \rho \in \mathcal{B},
$$

where $\log _{G}(x)$ is a group logarithm of the form (5).

As an immediate consequence of Theorem 1, we have the following result.

Theorem 2. A trace-norm group negativity $L_{G}(\rho)=\log _{G}\left\|\rho^{\Gamma}\right\|_{1}$ associated with a subadditive group logarithm satisfies the monotonicity property:

$$
\sum_{i} p_{i} L_{G}\left(\rho_{i}\right) \leqslant L_{G}(\rho) .
$$

Proof. It suffices to assume $p=1$ in the previous discussion. In particular, we have identically $c(1)=1$ and $k(1)=0$ into Eq. (17).

The trace-norm group negativities can be regarded as a new quantum version of the $Z$-entropies introduced in [37]. The main novelty of the present construction is that the functional (22) is the trace-norm of the partial transposition of a quantum state whose spectrum need not be, in general, a probability distribution.

Proposition 2. Any trace-norm group negativity is positive semi-definite over the space $\mathcal{B}$ of bounded linear operators of a Hilbert space $\mathcal{H}$ and vanishes for states with positive partial transpose. Furthermore, it is strictly composable: if $\mathcal{H}=\mathcal{H}_{1} \otimes \mathcal{H}_{2}$ then

$$
L_{G}(\sigma \otimes \tau)=\Phi\left(L_{G}(\sigma), L_{G}(\tau)\right)
$$

for any pair of states $\sigma \in \mathcal{B}_{1}$ and $\tau \in \mathcal{B}_{2}$ where $\Phi(x, y)=G\left(G^{-1}(x)+G^{-1}(y)\right)$.

Proof. Since $\operatorname{tr} \rho=\operatorname{tr} \rho^{\Gamma}$, it follows that $\left\{\rho^{\Gamma}\right\}_{-}=0$ only when $\left\|\rho^{\Gamma}\right\|_{1}=1$. Definition 10 implies that $L_{G}(\rho)=0$ in this case, whereas $L_{G}(\rho)>0$ in the other cases (being $\log _{G}$ strictly increasing). Composability is assured by the functional equation associated with the group logarithm $\log _{G}(x)=G(\ln x)$.

In our framework, the original logarithmic negativity [43] corresponds to the choice $G(t)=t$ which leads to the additive group $\Phi(x, y)=x+y$. A new nontrivial example is provided by the use of the $q$-logarithm of Eq. (7).

Definition 11. The trace-norm q-negativity of a state $\rho \in \mathcal{B}$ is the function

$$
L^{(q)}(\rho):=\frac{\left\|\rho^{\Gamma}\right\|_{1}^{1-q}-1}{1-q}, \quad q>1 .
$$

For $q \rightarrow 1$, it reduces to the logarithmic negativity $\lim _{q \rightarrow 1} L^{(q)}(\rho)=L(\rho)$. Furthermore, for any pair $\sigma \in \mathcal{B}_{1}$ and $\tau \in \mathcal{B}_{2}$ it follows

$$
L^{(q)}(\sigma \otimes \tau)=L^{(q)}(\sigma)+L^{(q)}(\tau)+(1-q) L^{(q)}(\sigma) L^{(q)}(\tau) .
$$

The trace-norm $q$-negativity is thus associated to the same composition law (called in algebraic topology the multiplicative formal group law [16]), namely $\Phi(x, y)=$ $x+y+(1-q) x y$ as both the classical and the quantum versions of the Tsallis entropy $[40,41]$. It is worth mentioning that the entanglement entropy associated 
to the Tsallis entropy (its evaluation over the reduced density matrix of a bipartite pure state) has been used in [42] to characterize the separability of a family of quantum states, correctly recovering Peres criterion for a concrete family of states.

Actually, Definitions 10 and 11 are naturally adapted to the Peres criterion [24], which can be applied to any state. Whenever the Peres criterion is necessary and sufficient, then $L_{(q)}(\rho)>0$ and, generally speaking, $L_{G}(\rho)>0$ for entangled states only. However, in a more general context (i.e. for higher dimensional spaces), $L_{G}(\rho)>0$ for NPT-entangled states only (here, by NPT-entangled states we mean quantum states with non-positive partial transpose).

Clearly, the trace-norm $q$-negativity suggests further generalization in terms of general entanglement witnesses [27], namely quantities that separate an entangled state from the set of separable ones in more general scenarios, whereas partial transpose separates in a necessary and sufficient way quantum systems associated to Hilbert spaces of dimension strictly lower than eight, namely when $\min _{i=1,2} \operatorname{dim} \mathcal{H}_{i}=2$ and $\max _{i=1,2} \operatorname{dim} \mathcal{H}_{i}=2,3$.

When $\operatorname{dim} \mathcal{H} \geqslant 8$, positivity of partial transposition is only necessary for separability and thus there exist entangled states with positive partial transpose. For all of them, $\left\{\rho^{\Gamma}\right\}_{-}=0$ and $L_{G}(\rho)=0$, in particular, when $G(t)=t$ one recovers the well-known fact that the logarithmic negativity vanishes $L(\rho)=0$ on PPT entangled states.

Remark 6. The fact that trace-norm group negativities are strictly composable is a non-trivial property, essentially related to their non-trace-form functional expression. Indeed, when dealing with standard entropies over a probability space, classical strict composability prevents the use of infinitely many trace-form entropies, namely functions of probability distributions $\left(p_{1}, \ldots, p_{W}\right)$ of the form $\sum_{i=1}^{W} f\left(p_{i}\right)$, $f(0)=f(1)=0$. Precisely, a theorem proved in [12] states, under mild hypotheses, that the most general trace-form entropy which is strictly composable is Tsallis entropy (recovering Boltzmann's entropy when $q \rightarrow 1$ ). Thus, using the more commonly adopted trace-form functionals one is lead to weakly composable group entropies [36], which are composable over the product of uniform distributions only. Instead, strictly composable entropies are allowed in the non-trace-form functional class. Indeed, each of the $Z$-entropies in Eq. (8) is strictly composable, with a specific composition law; thus, one can associate a trace-norm group negativity with each of them. The first of such pairs is represented by the original Tsallis $q$-entropy and the trace-norm $q$-negativity of Definition 11.

To summarize, the group norms for $p=1$ possess more regularity properties (they are entanglement monotones) and can be used as kind of nonadditive entanglement witnesses.

To complete the discussion, we shall focus on the additive case for $p \geqslant 1$.

\section{P-NORM ADDITIVE NEGATIVITY AS AN UPPER BOUND FOR DISTILLABILITY}

A crucial property of the additive $p$-norm (11) introduced in the present work is the fact that it represents an upper bound for distillability. In our analysis, we shall closely follow the notation and the discussion of Ref. [43].

We define $\mathcal{L}_{p}(\Omega):=\ln \|\Omega\|_{p}=\frac{1-p}{p} \ln N$, where $\Omega$ is the (diagonal) density matrix of the maximally mixed (separable) state. Then, we can introduce the normalized 
p-norm negativity

$$
\tilde{\mathcal{L}}_{p}(\rho):=\mathcal{L}_{p}(\rho)-\mathcal{L}_{p}(\Omega) .
$$

Note that the standard (trace-norm) logarithmic negativity is already normalized:

$$
\tilde{\mathcal{L}}_{1}(\rho)=L(\rho) .
$$

In a completely analogous way, one can normalize any $p$-norm group negativity.

Assume that we have a bipartite state $\rho$ and multiple copies of it obtained by means of LOCC. We recall that its distillation rate is the best rate at which we can extract near-perfect singlet states from its copies. In particular, given a large number of copies of the state, its asymptotic distillation rate is called its entanglement of distillation $E_{D}(\rho)$.

Let us consider $n_{\alpha}$ copies of $\rho$ and let $Y$ be a maximally entangled state of two qubits. Then, we are interested in the best approximation to $m_{\alpha}$ copies of $Y$ that can be obtained from $\rho^{\otimes n_{\alpha}}$ by means of LOCC.

We introduce [43]

$$
\Delta\left(Y^{\otimes m_{\alpha}, \rho^{\otimes n_{\alpha}}}\right)=\inf _{\mathscr{P}}\left\|Y^{\otimes m_{\alpha}}-\mathscr{P}\left(\rho^{\otimes n_{\alpha}}\right)\right\|_{1} .
$$

Here $\mathscr{P}$ runs over all deterministic protocols obtained from LOCC.

We say that $c$ is an achievable distillation rate for $\rho$, if for any sequences $n_{\alpha}$, $m_{\alpha} \rightarrow \infty$ of integers such that $\lim \sup _{\alpha}\left(n_{\alpha} / m_{\alpha}\right) \leqslant c$ we have

$$
\lim _{\alpha} \Delta\left(Y^{\otimes m_{\alpha}, \rho^{\otimes n_{\alpha}}}\right)=0 .
$$

Thus, the distillable entanglement is the supremum of all achievable distillation rates. If we allow a small error level, we can introduce the distillable entanglement at error level $\epsilon$, denoted by $E_{D}^{\epsilon}(\rho)$, which is characterized by the weaker condition

$$
\lim _{\alpha} \Delta\left(Y^{\otimes m_{\alpha}, \rho^{\otimes n_{\alpha}}}\right) \leqslant \epsilon .
$$

In this context, our main result is the following

Theorem 3. Let $\tilde{\mathcal{L}}_{p}(\rho)$ be the normalized logarithmic p-norm negativity. Then, for any $p \geqslant 1$ we have

$$
\tilde{\mathcal{L}}_{p}(\rho) \geqslant E_{D}^{\epsilon} .
$$

Proof. As is well known [43], the standard logarithmic negativity satisfies the upper bound

$$
L(\rho) \geqslant E_{D}^{\epsilon} .
$$

We also remind the inequalities $(p>1)$

$$
\|\cdot\|_{p} \leqslant\|\cdot\|_{1} \leqslant N^{1-1 / p}\|\cdot\|_{p} .
$$

Consequently, from the first inequality (30), we get

$$
\mathcal{L}_{p}(\rho) \leqslant L(\rho) .
$$

From the second one, we have

$$
L(\rho) \leqslant-\mathcal{L}_{p}(\Omega)+\mathcal{L}_{p}(\rho) .
$$

Thus, due to inequality (29), we get

$$
\mathcal{L}_{p}(\rho)-\mathcal{L}_{p}(\Omega) \geqslant E_{D}^{\epsilon} .
$$


Using Definition 24, we conclude that

$$
\tilde{\mathcal{L}}_{p}(\rho) \geqslant E_{D}^{\epsilon} .
$$

\section{Future Perspectives}

As we have shown, group theory offers a natural way to generalize the notion of negativity. This work represents a first exploration of a new, infinite class of easily computable entropic-type measures of entanglement. We have introduced the $p$-norm and trace-norm group negativities and focused on the mathematical study of some of the main analytical properties of the class. Our main result is the construction of a large family of composable entanglement monotones.

Several aspects of the theory deserve further analysis. It is clear that composability is crucial in order to compute entanglement entropy of bipartite or multipartite systems in a natural way, starting from the knowledge of the entropy of its constituents. As we suggested, such a property is fundamental to study distillable entanglement. Therefore, an interesting open problem is to ascertain if all of the group-theoretical negativities introduced here, apart the logarithmic $p$-norm negativity, can provide upper or lower bounds to the asymptotic distillation rate by means of LOCC, when we consider a large number of copies of the state $\rho^{\otimes n_{\alpha}}$.

At the same time, it would be very interesting to apply the large family of entropic functionals introduced in this work in the study of finite temperature systems in conformal field theories [7]. From this point of view, one-parametric (or multi-parametric) entanglement monotones could play a role similar to that played by Rényi's entropy in the case of the entanglement detection of the ground state of one-dimensional many body systems, and in the study of their criticality properties [5].

We wish to point out that the language of formal group theory can be directly related to the study of alternative formulations of both classical and quantum mechanics. Indeed, as shown in [13], the linear structure of the theory can be replaced by a non-additive structure generated by means of a suitable diffeomorphism, which would play the same role as the group logarithm of the present theory. In particular, this perspective opens the possibility of performing non-equivalent Weyl quantizations of physical systems, circumventing the von Neumann uniqueness theorem. The generalized negativities introduced in the present work could play a significant role in these alternative formulations. We shall discuss these aspects in detail elsewhere.

Another interesting problem is to give an interpretation of both group negativities and the quantum version of the relative entropies (9) within the context of quantum information geometry, especially in connection with the problem of tomographic reconstruction of quantum metrics [1], [2], [23], [10]. Also, an intriguing problem is to establish a connection between the approach proposed in this article and that one developed independently in [44].

Finally, we also plan to apply generalized negativities to the study of entanglement properties of some concrete examples of quantum systems, in particular integrable spin chains of Haldane-Shastry type [14] [15].

Work is in progress along these lines. 


\section{ACKNOWLEDGEMENT}

The authors wish to thank the anonimous referees for useful suggestions, which improved the readability of the article.

J.C. would like to thank Aleksander M. Kubicki for several enlightening discussions.

G.M. would like to thank the support provided by the Santander/UC3M Excellence Chair Programme 2019/2020.

The research of P.T. has been supported by the research project PGC2018094898-B-I00, Ministerio de Ciencia, Innovación y Universidades and Agencia Estatal de Investigación, Spain, and by the Severo Ochoa Programme for Centres of Excellence in R\&D (CEX2019-000904-S), Ministerio de Ciencia, Innovación y Universidades y Agencia Estatal de Investigación, Spain.

G. M and P. T. are members of the Gruppo Nazionale di Fisica Matematica (INDAM), Italy.

\section{APPENDIX:FORMAL GROUPS AND FORMAL RINGS}

Let $R$ be a commutative associative ring with identity, and $R \llbracket x_{1}, x_{2}, \ldots \rrbracket$ be the ring of formal power series in the variables $x_{1}, x_{2}, \ldots$ with coefficients in $R$. We shall assume that $R$ is torsion-free.

Definition 12. [4] A commutative one-dimensional formal group law over $R$ is a formal power series $\Phi \in R \llbracket x, y \rrbracket$ such that

(1) $\Phi(x, 0)=\Phi(0, x)=x$

(2) $\Phi(\Phi(x, y), z)=\Phi(x, \Phi(y, z))$.

The formal group law is said to be commutative if $\Phi(x, y)=\Phi(y, x)$.

Observe that the existence of an inverse formal series $\varphi \in R \llbracket x \rrbracket$ such that $\Phi(x, \varphi(x))=0$ is a direct consequence of the previous definition. This justifies the "group" terminology for these algebraic structures.

Let $B=\mathbf{Z} \llbracket b_{1}, b_{1}, \ldots \rrbracket$ and consider the following series in $B \llbracket s \rrbracket$

$$
F(s)=s+\sum_{i=1}^{\infty} b_{i} \frac{s^{i+1}}{i+1} .
$$

If $G \in B \llbracket t \rrbracket$ is its compositional inverse (namely $F(G(t))=t$ and $G(F(s))=s$ ), one has

$$
G(t)=t+\sum_{k=1}^{\infty} a_{k} \frac{t^{k+1}}{k+1}
$$

with $a_{1}=-b_{1}, a_{2}=\frac{3}{2} b_{1}^{2}-b_{2}, \ldots$. Given the formal power series $F$ and $G$ as in Eqs. (34), the Lazard formal group law [16] is defined by the formal power series

$$
\Phi_{\mathrm{L}}\left(s_{1}, s_{2}\right)=G\left(G^{-1}\left(s_{1}\right)+G^{-1}\left(s_{2}\right)\right)
$$

whose coefficients, generate over $\mathbf{Z}$ a subring $L \subset B \otimes \mathbf{Q}$. In other words, the Lazard ring is defined over a subring of the original ring $B \otimes \mathbf{Q}$, called the Lazard ring.

For any commutative one-dimensional formal group law over any ring $R$, there exists a unique homomorphism $L \rightarrow R$, under which the Lazard group law is mapped into the given group law. This is called the universal property of the Lazard 
group. Also, it is important to notice that for any commutative one-dimensional formal group law $\Phi(x, y)$ over $R$, there exists a series $\phi(x)$ such that

$$
\phi(x)=x+O\left(x^{2}\right), \quad \text { and } \Phi(x, y)=\phi^{-1}(\phi(x)+\phi(y)) .
$$

Finally, let us also define the notion of formal ring (see [9]).

Definition 13. Let $(R,+, \cdot)$ be a unital ring. A formal ring is a triple $(R, \Phi, \Psi)$ where $\Phi, \Psi \in R \llbracket x, y \rrbracket$ are formal power series such that

(1) $\Phi$ is a commutative formal group law.

(2) $\Psi$ satisfies the relations

$$
\begin{aligned}
& \Psi(\Psi(x, y), z)=\Psi(x, \Psi(y, z)) \\
& \Psi(x, \Phi(y, z))=\Phi(\Psi(x, y), \Psi(x, z)) \\
& \Psi(\Phi(x, y), z)=\Phi(\Psi(x, z), \Psi(y, z)) .
\end{aligned}
$$

The formal ring will be said to be commutative if $\Psi(x, y)=\Psi(y, x)$.

\section{REFERENCES}

[1] S. I. Amari, H. Nagaoka. Methods of information geometry, Vol. 191, American Mathematical Society (2000).

[2] S. I. Amari. Information geometry and its applications, Applied Mathematical Sciences. Japan: Springer (2016).

[3] L. Amico, R. Fazio, A. Osterloh and V. Vedral. Entanglement in many-body systems, Rev. Mod. Phys. 80, 517 (2008).

[4] S. Bochner. Formal Lie groups, Ann. Math. 47 (1946), 192-201.

[5] P. Calabrese and J. Cardy. Entanglement entropy and quantum field theory, J. Stat. Mech. P06002 (2004)

[6] P Calabrese, J. Cardy and E. Tonni. Entanglement negativity in quantum field theory, Phys. Rev. Lett. 109 (13), 130502 (2012).

[7] P. Calabrese, J. Cardy and E. Tonni. Finite temperature entanglement negativity in conformal field theory, J. Phys. A: Math. Theor. 48 (1), 015006 (2014)

[8] J. Carrasco, F. Finkel, A. Gonzalez-Lopez, and P. Tempesta. A duality principle for the multi-block entanglement entropy of free fermion systems, Nature - Scient. Rep. 7 (1), 11206 (2017).

[9] J. Carrasco, P. Tempesta. Formal rings, arxiv: 1902.03665 (2019).

[10] F. M. Ciaglia, F. Di Cosmo, M. Laudato, G. Marmo, F. M. Mele, F. Ventriglia, P. Vitale. A pedagogical intrinsic approach to relative entropies as potential functions of quantum metrics: the q-z Family, Ann. Phys. 395 (2018).

[11] V. Eisler, Z. Zimbors. Entanglement negativity in two-dimensional free lattice models, Phys. Rev. B 93 (11), 115148 (2016).

[12] A. Enciso, P. Tempesta. Uniqueness and characterization theorems for generalized entropies, J. Stat. Mech. 123101 (2017).

[13] E. Ercolessi, A. Ibort, G. Marmo and G. Morandi. Alternative linear structures for classical and quantum systems, Int. J. Mod. Physics A 22, 3039-3064 (2007).

[14] F. D. M. Haldane. Exact Jastrow-Gutzwiller resonating-valence-bond ground state of the spin-1/2 antiferromagnetic Heisenberg chain with $1 / r^{2}$ exchange. Phys. Rev. Lett. 60635 (1988).

[15] B. S. Shastry. Exact solution of an $S=1 / 2$ Heisenberg antiferromagnetic chain with longranged interactions. Phys. Rev. Lett. 60639 (1988).

[16] M. Hazewinkel. Formal Groups and Applications, Academic Press, New York (1978).

[17] M. Horodecki. Entanglement measures, Quantum Inf. Comp. 1, 3 (2001).

[18] M. Horodecki, P. Horodecki, R. Horodecki. Separability of mixed states: necessary and sufficient conditions, Phys. Lett. A 223, 1 (1996).

[19] P. Horodecki and R. Horodecki. Distillation and bound entanglement, Quantum Inf. Comp. 1, 45 (2001). 
[20] V. Ilic and M. Stankovic, Generalized Shannon-Khinchin axioms and uniqueness theorem for pseudo-additive entropies, Physica A 411, 138-145 (2014).

[21] P. Jizba and J. Korbel, Maximum Entropy Principle in statistical inference: case for nonShannonian entropies, Phys. Rev. Lett. 122, 120601 (2019).

[22] A. I. Khinchin. Mathematical Foundations of Information Theory, Dover, New York (1957).

[23] V. I. Man'ko, G. Marmo, F. Ventriglia, P. Vitale. Metric on the space of quantum states from relative entropy. Tomographic reconstruction, J. Phys. A: Math. Theor. 50, 335302 (2017).

[24] A. Peres. Separability Criterion for Density Matrices, Phys. Rev. Lett. 77, 1413 (1996).

[25] M. B. Plenio. Logarithmic negativity: A full entanglement monotone that is not convex, Phys. Rev. Lett. 95, 090503 (2005).

[26] M. B. Plenio and V. Vedral. Teleportation, entanglement and thermodynamics in the quantum world, Contemp. Phys. 39, 431 (1998).

[27] M. B. Plenio and S. Virmani. An introduction to entanglement measures, Quantum Inf. Comp. 7, 001051 (2007).

[28] M. A. Rodríguez, A. Romaniega and P. Tempesta, A new class of entropic information measures, formal group theory and information geometry, Proc. Royal Soc. A 475, 20180633 (2019).

[29] P. Ruggiero, V. Alba and P. Calabrese. Entanglement negativity in random spin chains, Phys. Rev. B 94 (3), 035152 (2016)

[30] J.-P. Serre. Lie algebras and Lie groups, Lecture Notes in Mathematics, 1500 Springer-Verlag, 1992.

[31] C. E. Shannon. A mathematical theory of communication, Bell Syst. Tech. J. 27 (1948) 379-423, 27 623-653 (1948).

[32] C. E. Shannon and W. Weaver. The mathematical Theory of Communication, University of Illinois Press, Urbana, USA (1949).

[33] P. Tempesta. L-series and Hurwitz zeta functions associated with the universal formal group, Annali Sc. Norm. Superiore, Classe di Scienze, IX, 1-12 (2010).

[34] P. Tempesta. Group entropies, correlation laws and zeta functions, Phys. Rev. E 84, 02112 (2011).

[35] P. Tempesta. The Lazard formal group, universal congruences and special values of zeta functions, Trans. Am. Math. Society, 367, 7015-7028 (2015).

[36] P. Tempesta. Beyond the Shannon-Khinchin formulation: The composability axiom and the universal group entropy. Ann. Phys. 365, 180-197 (2016).

[37] P. Tempesta. Formal Groups and Z-Entropies, Proc. Royal Soc. A, Vol. 472, 20160143 (2016).

[38] P. Tempesta, Multivariate group entropies, super-exponentially growing complex systems, and functional equations, Chaos 30, 123119 (2020).

[39] P. Tempesta and H. J. Jensen, Universality Classes and Information-Theoretic Measures of Complexity via Group Entropies, Nature - Sci. Rep. 10, 5952 (2020).

[40] C. Tsallis. Possible generalization of the Boltzmann-Gibbs statistics, J. Stat. Phys. 52, Nos. 1/2, 479-487 (1988).

[41] C. Tsallis. Introduction to Nonextensive Statistical Mechanics-Approaching a Complex World, Springer, Berlin (2009).

[42] C. Tsallis, S. Lloyd and M. Baranger. Peres criterion for separability through nonextensive entropy, Phys. Rev. A 63, 042104.

[43] G. Vidal and R. F. Werner. Computable measures of entanglement, Phys. Rev. A 65, 132314 (2002).

[44] X. Wang and M. Wilde. $\alpha$-logaritmic negativity, Phys. Rev. A 102, 032416 (2020). 
Institut für Theoretische Physik, Universität Innsbruck, Innrain 52, 6020 Innsbruck, Austria

Email address: joseacar@ucm.es

Dipartimento di Fisica, Università Federico II and Sezione INFN di Napoli, Via Cintia, NAPOLI, ITALY

Email address: marmo@na.infn.it

Instituto de Ciencias Matemáticas, C/ Nicolás Cabrera, No 13-15, 28049 Madrid, Spain, and Departamento de Física Teórica, Facultad de Ciencias Físicas, Universidad Complutense de Madrid, 28040 - Madrid, Spain

Email address: p.tempesta@fis.ucm.es, piergiulio.tempesta@icmat.es 